\title{
Total Flavonoids from Leaves of Carya Cathayensis Ameliorate Renal Fibrosis via the miR-21/Smad7 Signaling Pathway
}

\author{
Xiaoli Wu $u^{a, b} \quad$ Xiaoqiang Ding ${ }^{c, d, e, f, g} \quad$ Zhishan Ding ${ }^{a} \quad$ Ping Jia ${ }^{c, d, e, f, g}$ \\ ${ }^{a}$ College of Pharmaceutical Science, Zhejiang Chinese Medical University, Hangzhou, ${ }^{\mathrm{b}}$ Traditional \\ Chinese Medicine Pharmacology Laboratory, Longhua Hospital, Shanghai University of Traditional \\ Chinese Medicine, Shanghai, 'Department of Nephrology, Zhongshan Hospital, Fudan University, \\ Shanghai, 'Shanghai Medical Center of Kidney, Shanghai, eShanghai Institute of Kidney and Dialysis,

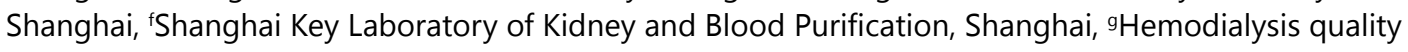 \\ control center of Shanghai, Shanghai, China
}

\section{Key Words}

Total flavonoid • Renal fibrosis • MicroRNA • Signaling pathway

\begin{abstract}
Background/Aims: Renal tubulointerstitial fibrosis is the most common pathway of progressive kidney injury, leading to end-stage renal disease. At present, no effective prophylactic treatment method is available. This study investigated the anti-fibrotic effects of total flavonoids (TFs) extracted from leaves of Carya Cathayensis in vivo and in vitro, and explored the underlying mechanisms. Methods: Anti-fibrotic effects of TFs were measured using a mouse model of unilateral ureteral obstruction (UUO) and in transforming growth factor- $\beta 1$ (TGF- $\beta 1$ )-treated mouse tubular epithelial cells (mTECs). mRNA expression and protein levels of Collagen I, Collagen III, and $\alpha$-smooth muscle actin $(\alpha-S M A)$ were also tested by realtime reverse transcription PCR and western blot analysis. To elucidate the underlying mechanisms, expression of miR-21 was examined in mTECs treated with TFs using miR-21 mimics transfected into mTECs before TGF- $\beta 1$ and TFs treatment. Regulation of mothers against decapentaplegic homolog (Smad) signaling by miR-21 was subsequently validated via overexpression and deletion of miR-21 followed by a luciferase assay. Results: TFs treatment attenuated renal fibrosis, and inhibited expression of collagens and $\alpha$-SMA in the kidneys of mice subjected to UUO. In vitro, the TFs significantly decreased expression of fibrotic markers in TGF- $\beta 1$ treated mTECs. Moreover, TFs reduced miR-21 expression in a time- and dose-dependent manner in mTECs, increased expression of Smad7, and decreased phosphorylation of Smad3. Treatment with miR-21 mimics abolished the anti-fibrotic effects of the TFs on the TGF- $\beta 1$ treated mTECs. In addition, genetic deletion of miR-21 upregulated expression of Smad7 and suppressed phosphorylation of Smad3, attenuating renal fibrosis in mice. Bioinformatics predictions revealed the potential binding site of miR-21 in the $3^{\prime}$-untranslated region of
\end{abstract}

Ping Jia

and Zhishan Ding
Department of Nephrology, Zhongshan Hospital, Fudan University

Shanghai (China)

E-Mail jia.ping1@zs-hospital.sh.cn; zjtcmdzs@163.com 


\section{Cellular Physiology Cell Physiol Biochem 2018;49:1551-1563 \\ \begin{tabular}{l|l|l} 
DOI: 10.1159/000493458 & a 2018 The Author(s). Published by S. Karger AG, Basel \\
www.karger.com/cpb
\end{tabular} \\ Wu et al.: Total Flavonoids Ameliorate Renal Fibrosis}

Smad7, and this was further confirmed by the luciferase assay. Conclusion: TFs ameliorate renal fibrosis via a miR-21/Smad7 signaling pathway, indicating a potential therapy for the prevention of renal fibrosis.

(C) 2018 The Author(s)

Published by S. Karger AG, Basel

\section{Introduction}

Carya cathayensis, family Juglandaceae, is widely distributed around the world. It is used as both food and medicine, possessing various biological activities. For example, its nut kernel [1] and shell [2] were found to have a variety of pharmacological effects, including anti-tumor, anti-inflammatory and anti-oxidative activity. Its leaves are also rich in flavonoids. In our previous study, we isolated total flavonoids (TFs) from C. cathayensis leaves, and then purified and identifiedthe chemical constituents [3]. They were found to be composed of five flavonoids, namely, cardamonin, wogonin, pinocembrin, chrysin, and pinostrobin chalcone. Moreover, each of these flavonoids and the compound TFs were able to inhibit vascular endothelial growth factor-induced angiogenesis and cell proliferation [3]. Recently, we further revealed that TFs from the leaves of $C$. cathayensis also protect against early development of atherosclerosis via inhibition of arterial medial smooth muscle cell proliferation and macrophage accumulation in arterial adventitia [4]. This led us to hypothesize that the TFs might also possess an anti-fibrotic effect.

Renal tubulointerstitial fibrosis, characterized by interstitial extracellular matrix (ECM) deposition and myofibroblast accumulation, is regarded as the common end point of chronic kidney disease (CKD) [5], leading to derangement of renal architecture and irreversible impairment of renal function [6]. It is well documented that transforming growth factor$\beta 1$ (TGF- $\beta 1$ )/mothers against decapentaplegic homolog (Smad) signaling is the central pathway regulating the development and progression of fibrosis $[7,8]$. TGF- $\beta 1$ binds to its specific receptor, stimulating Smad2/Smad3 phosphorylation, which subsequently results in the formation of complexes with Smad4. These complexes are then translocated to the nuclei, where they regulate expression of various TGF- $\beta 1$ target genes, such as collagens $[9,10]$. In addition, Smad 3 can bind directly to promoters of collagens, namely, $\alpha$-smooth muscle actin $(\alpha$-SMA $)$ and fibronectin, thereby regulating their expression $[7,11]$. Smad7, an inhibitor of Smad, is a negative regulator of TGF- $\beta 1 /$ Smad signaling, suppressing phosphorylation of Smad2/Smad3, promoting TGF- $\beta 1$ receptor type I degradation $[7,12]$, and consequently inhibiting fibrogenesis. Therapeutic strategies targeting TGF- $\beta 1 / \mathrm{Smad}$ signaling could therefore effectively prevent renal fibrosis.

MicroRNAs (miRNAs) are small (20-23 nucleotides) non-coding RNAs that function mainly to suppress target gene expression via translation inhibition or mRNA degradation [13]. Dysregulated miRNA expression has been observed in a number of human diseases and in animal models. Emerging evidence suggests that various pathophysiological processes are regulated by miRNAs, including tissue fibrosis [14]. Among the fibrosis-related miRNAs, miR-21 has been found to play an important role in promoting fibrogenesis in the kidney [15-17], heart [18, 19], liver [20] and lung [21]. Moreover, miR-21 is one of the most highly upregulated miRNAs during kidney injury, both acute kidney injury [22] and CKD [17]. In our previous study, we revealed that miR-21 was up-regulated soon after ischemia in a mouse model of renal ischemia reperfusion injury [23]. Moreover, when renal tubulointerstitial fibrosis appears, miR-21 expression is maintained at a high level. miR-21 is significantly upregulated in mice kidneys in response to unilateral ureteral obstruction (UUO). Inhibition of miR-21 was found to attenuate renal fibrosis in two well-established animal models [1517]. These findings suggest that miR-21 could play a novel therapeutic role in the prevention of renal fibrosis.

In this study, we examined the effects of TFs on renal fibrosis in vitro and in vivo, revealing a decrease in miR-21 expression in TGF- $\beta 1$-treated mouse tubular epithelial cells (mTECs) and in kidneys of mice subjected to UUO. Moreover, up-regulation of Smad7 expression and inhibition of Smad3 phosphorylation was also observed, thereby attenuating renal fibrosis. 
Smad7 was therefore confirmed as the target gene of miR-21 in tubular epithelial cells (TECs). Meanwhile, overexpression of miR-21 abolished the anti-fibrotic effects of the TFs on renal fibrosis. Overall, these findings suggest that the TFs ameliorate renal fibrosis via the miR-21/Smad7 signaling pathway.

\section{Materials and Methods}

\section{Cell culture and treatment}

mTECs were purchased from Caltag Medsystems (Buckingham, UK) and cultured in RPMI-1640 medium (Gibco, Carlsbad, CA) supplemented with $10 \%$ fetal bovine serum in a humidified incubator at $37^{\circ} \mathrm{C}$ with $5 \% \mathrm{CO}_{2}-95 \%$ air TFs were isolated from C. cathayensis leaves, and then purified and identified, as described in our previous study [3]. TFs samples $(5 \mathrm{mg}$ ) were dissolved in $1 \mathrm{~mL}$ dimethyl sulfoxide (DMSO) to create a stock solution $(5 \mathrm{mg} / \mathrm{mL}$ ), which was serially diluted in RPMI1640 medium to give final concentrations for the in vitro experiment. The TFs were added to the cells at concentrations of $0,5,10,20$, 40 , and $80 \mu \mathrm{g} / \mathrm{mL}$ for $24 \mathrm{~h}$, and the cell viability was assayed. According to the results, $10 \mu \mathrm{g} / \mathrm{mL} \mathrm{TFs} \mathrm{was}$ selected for subsequent analysis, in which they were added to the cells for $0,2,6,12,24$, and $48 \mathrm{~h}$. In an additional experiment, cells were stimulated with recombinant human TGF- $\beta 1$ (R\&D Systems, Minneapolis, $\mathrm{MN}$ ) at a concentration of $10 \mathrm{ng} / \mathrm{mL}$ for $24 \mathrm{~h}$ with and without the TFs.

\section{Flow cytometry and cell viability assay}

mTECs were treated with TFs at indicated concentrations. Fluorescein isothiocyanate-conjugated AnnexinV/propidium iodide (PI) double staining was applied to assay cell apoptosis using a flow cytometer (Altra; Beckman Coulter, Inc., Brea, CA). Quadrant statistics on AnnexinV-positive/PI-negative, double positive cells, AnnexinV-negative/PI-positive, and double negative cells were utilized.

The viability of mTECs treated with and without TFs was assessed via the 3-(4, 5-dimethylthiazol-2-

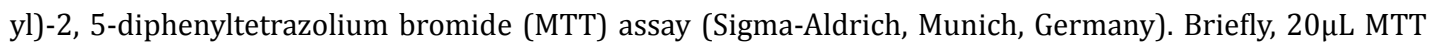
solution (5mg/mL; Sigma-Aldrich) was added to each well of a 96-well plate. After 4-h incubation, $200 \mu \mathrm{L}$ of DMSO was added to dissolve the formazan crystals. Absorbance at a wave length of $490 \mathrm{~nm}$ was measured on a microplate reader. All experiments were performed in triplicate.

\section{Mouse model of UUO and treatment}

C57BL/6 mice (Animal Center of Fudan University, Shanghai, China), miR-21\% mice (B6.129Mir21atm1Smoc, Shanghai Biomodel Organism Science \& Technology Development Co. Shanghai, China) and wild-type littermates, all 8-10 weeks old, were used in the mouse model. All animal experiments were approved by the Institutional Animal Care and Use Committee of Fudan University. The murine UUO model was as follows: after anesthesia with pentobarbital sodium $(50 \mathrm{mg} / \mathrm{kg}$, i.p.), the abdominal cavity was exposed via a midline incision. The left ureter was then isolated and ligated with 4-0 silk at the ureteropelvic junction. The contralateral kidney with ureter not ligated was used as a control. To examine the effect of TFs on renal fibrosis, TFs in $300 \mu \mathrm{L}$ DMSO were administered daily via oral-gastric syringe feeding from day 0 to day 13 after surgery. A dose of $50 \mathrm{mg} / \mathrm{kg}$ was selected based on our previous study [4]. An equivalent amount of DMSO was administered to the control group. Mice were sacrificed at day 14 after UUO.

\section{Masson's trichrome assay and immunohistochemistry}

Kidney slices were fixed in $10 \%$ formalin and embedded in paraffin. Sections $(5 \mu \mathrm{m}$ thick) were stained with Masson's trichrome and evaluated under light microscopy by a nephropathologist blinded to the experiments.

Immunohistochemistry was performed on the paraffinized sections as previously described [24] using the following antibodies: Collagen I, Collagen III, and $\alpha$-SMA (Abcam, Cambridge, MA). For semi-quantitative analysis, accumulation of Collagen I-, Collagen III-, and $\alpha$-SMA-positive cells was determined using a quantitative image analysis system (AxioVision 4, Carl Zeiss, Jena, Germany), as previously described [25]. 


\section{Cellular Physiology Cell Physiol Biochem 2018;49:1551-1563 \begin{tabular}{l|l|l}
\hline DOI: 10.1159/000493458 & $\begin{array}{l}\text { C) 2018 The Author(s). Published by S. Karger AG, Basel } \\
\text { www.karger.com/cpb }\end{array}$ \\
\hline
\end{tabular}}

Wu et al.: Total Flavonoids Ameliorate Renal Fibrosis

\section{Transient transfection with miRNAs}

Synthesized miR-21 mimics (GenePharma, Shanghai, China) were transfected into mTECs at a final concentration of $100 \mathrm{nM}$ using Lipofectamine 2000 (Invitrogen, Carlsbad, CA) mixed in Opti-MEM ${ }^{\circledR}$ I reduced serum medium (Gibco). An equal concentration of negative control oligonucleotides was used as a control. After transfection for $48 \mathrm{~h}$, the cells were stimulated with $10 \mathrm{ng} / \mathrm{mL}$ recombinant human TGF- $\beta 1$ for $24 \mathrm{~h}$ with and without TFs.

\section{Enzyme-linked immunosorbent assay}

The kidney tissue was homogenized in $1 \mathrm{~mL}$ phosphate-buffered saline and an enzyme-linked immunosorbent assay (ELISA) was performed for TGF- $\beta 1$ (Abcam) according to the manufacturer's instructions.

\section{Real-time reverse transcription PCR}

Total RNA was extracted from cells and dissected kidney tissue using TRIzol reagent (Invitrogen), and was reverse-transcribed to complementary DNA (PrimeScript RT reagent Kit; TaKaRa, Kyoto, Japan). Then, real-time reverse transcription PCR (qRT-PCR, SYBR Premix Ex TaqTM TaKaRa) was performed. Primers (Sangon, Shanghai, China) were as follows:

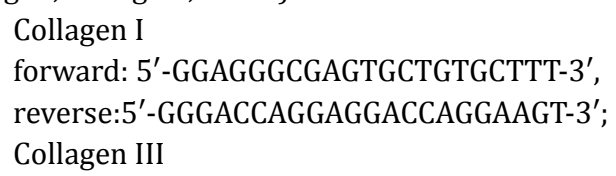

In addition, mature miR-21 was detected by TaqMan Real-Time Reverse Transcription-PCR using a specific miRNA probe and PCR master mix (Applied Biosystems, Hayward, CA) as described previously [26]. U6 snRNA and $\beta$-actin mRNA were used as internal controls for miR-21 and mRNA, respectively. The $2^{-\Delta \Delta C t}$ method was used to calculate fold changes, and relative gene levels were expressed as ratios to the control.

\section{Western blot}

Western blot analysis was performed as previously described [27] using the following antibodies: Collagen I, Collagen III, and $\alpha$-SMA (Abcam), Smad3 and p-Smad3 (Cell Signaling Technology, Danvers, MA), and Smad7 and $\beta$-actin (Santa Cruz Biotechnology, Santa Cruz, CA).

\section{Luciferase reporter assay}

The wild-type 3 -untranslated region (UTR) of Smad7 mRNA containing the putative miR-21 binding sequence or the mutated $3^{\prime}$-UTR fragment was cloned into a firefly luciferase reporter construct and co-transfected into mTECs with either the miR-21 mimic (100 nM), miR-NC $(100 \mathrm{nM})$, or vehicleusing Lipofectamine 2000 (Invitrogen). After 48-h transfection, luciferase activity was detected using the DualLuciferase Reporter Assay System (Promega, Madison, WI).

\section{Statistical analysis}

SPSS Version 16.0 software (SPSS Inc., Chicago, IL) was used for all statistical analysis. Data are presented as the mean \pm standard error of the mean. Student's t-tests were used for comparisons between two groups. For multiple group comparisons, one-way analysis of variance followed by the Bonferroni posthoc test was applied. A $p$-value $<0.05$ was considered statistically significant. 


\section{Cellular Physiology Cell Physiol Biochem 2018;49:1551-1563 \begin{tabular}{l|l} 
and Biochemistry & DOI: 10.1159/000493458 \\
Published online: 13 September, 2018 & $\begin{array}{l}\text { O } 2018 \text { The Author(s). Published by S. Karger AG, Basel } \\
\text { www.karger.com/cpb }\end{array}$
\end{tabular}}

\section{Results}

TFs treatment attenuated renal tubulointerstitial fibrosis in UUO mice

At day 14 after UUO,the ligated kidneys showed typical histologic features of tubulointerstitial fibrosis, characterized as widened interstitial space, obvious tubular dilatation, tubular atrophy and collagen matrix deposition, as revealed by Masson's trichrome staining (Fig. 1A). TFs treatment (TF-UUO group) significantly attenuated morphologic injury and reduced collagen matrix deposition in UUO kidneys, while administration of a vehicle control (Con-UUO group) had no effect. Next, we examined expression of fibrotic markers in the UUO mice, revealing enhanced accumulation of Collagen I, Collagen III, and $\alpha$-SMApositive cells in the ligated kidneys compared with normal kidneys (Fig. 1B, C). Consistent with this accumulation, qRT-PCR analysis revealed a marked increase in expression of

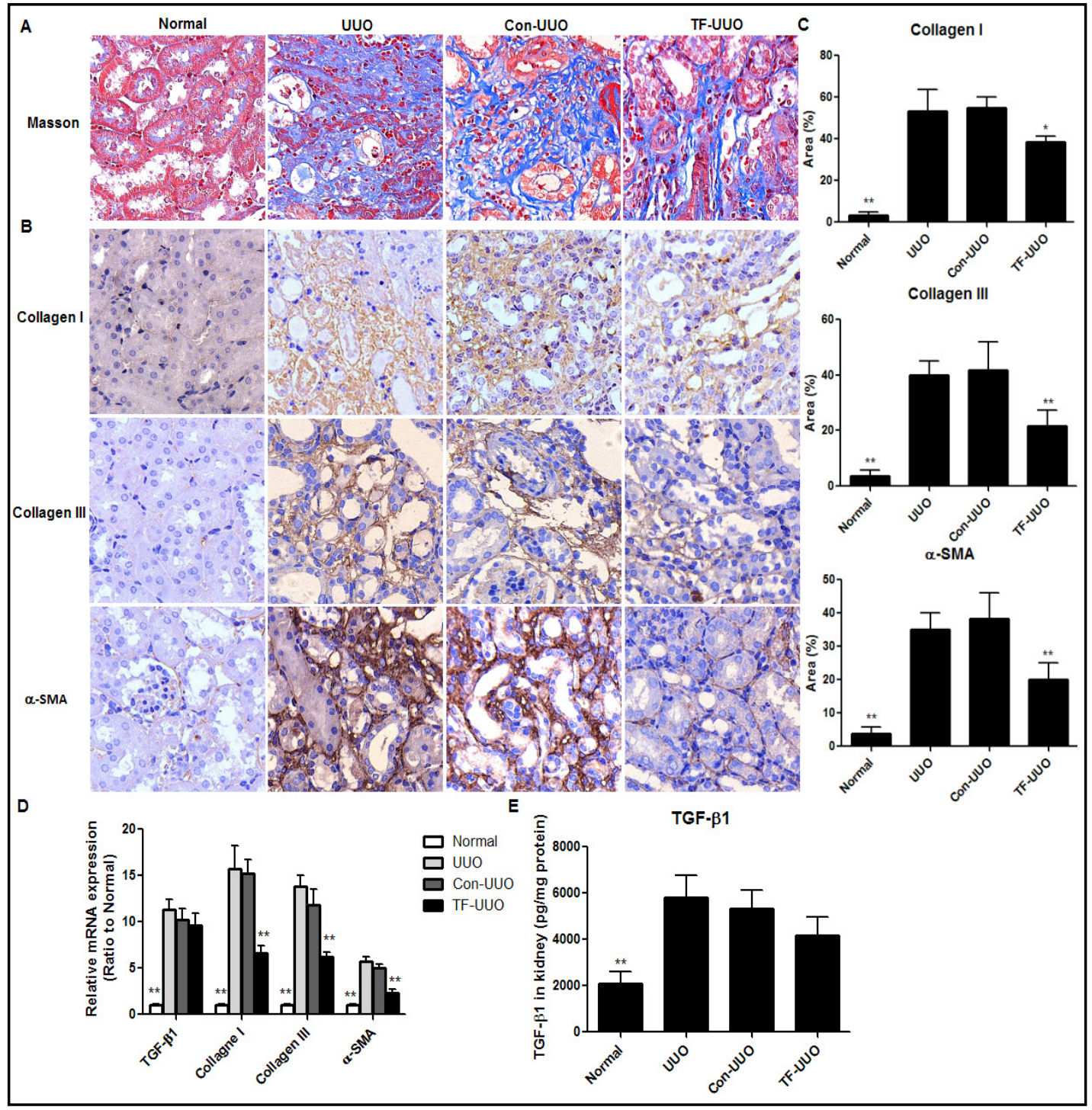

Fig. 1. TFs attenuated renal tubulointerstitial fibrosis in mice with UUO. (A) Masson trichrome staining of normal kidneys and UUO kidneys in mice treated with a vehicle control or TFs for 14 days after surgery. Magnification:×100. (B) Immunohistochemical staining of Collagen I, Collagen III, and $\alpha$-SMA. (C) Quantitative analysis of immunohistochemical staining. (D) Expression of TGF- $\beta 1$ mRNA, Collagen I mRNA, Collagen III mRNA, and $\alpha$-SMA mRNA. (E) ELISA analysis of TGF- $\beta 1$ protein in the kidneys. ${ }^{*} \mathrm{p}<0.05,{ }^{* *} \mathrm{p}<$ 0.01 , compared with the UUO group. 
collagen I, collagen III, and $\alpha$-SMA in the kidneys at day 14 after UUO (Fig. 1D). However, the accumulation of these fibrotic markers was significantly reduced after TFs treatment, and accordingly, expression was markedly decreased. These findings suggest that TFs can attenuate renal tubulointerstitial fibrosis. In addition, TGF- $\beta 1$ expression was significantly up-regulated in the kidneys of mice subjected to UUO. Interestingly, TFs treatment had no effect on TGF- $\beta 1$ expression in both mRNA and protein levels (Fig. 1D, E).

Effect of TFs on cell apoptosis, viability, and miR-21 expression in mTECs

To evaluate the effects of TFs on cell apoptosis and viability, mTECs were treated with different concentrations of TFs. TFs at a concentration of $20 \mu \mathrm{g} / \mathrm{mL}$ had no obvious effect on apoptosis and cell viability, whereas $40 \mu \mathrm{g} / \mathrm{mL}$ or more increased apoptosis to a small extent, thereby affecting cell viability (Fig. $2 \mathrm{~A}-\mathrm{C}$ ). These findings suggest that concentrations of $\leq 20$ $\mu \mathrm{g} / \mathrm{mL}$ have no cytotoxicity.

It is well known that miR-21 is one of the most important miRNAs during renal fibrosis. To determine whether TFs modulate miR-21 expression in mTECs, we analyzed expression of miR-21 in mTECs treated with different concentrations of TFs, and with $10 \mu \mathrm{g} / \mathrm{mL}$ TFs for $2 \mathrm{~h}, 6 \mathrm{~h}, 12 \mathrm{~h}, 24 \mathrm{~h}$, or $48 \mathrm{~h}$, TFs treatment down-regulated miR-21 expression in a dosedependent manner and significantly reduced miR-21 expression at a concentration of $5 \mu \mathrm{g} /$

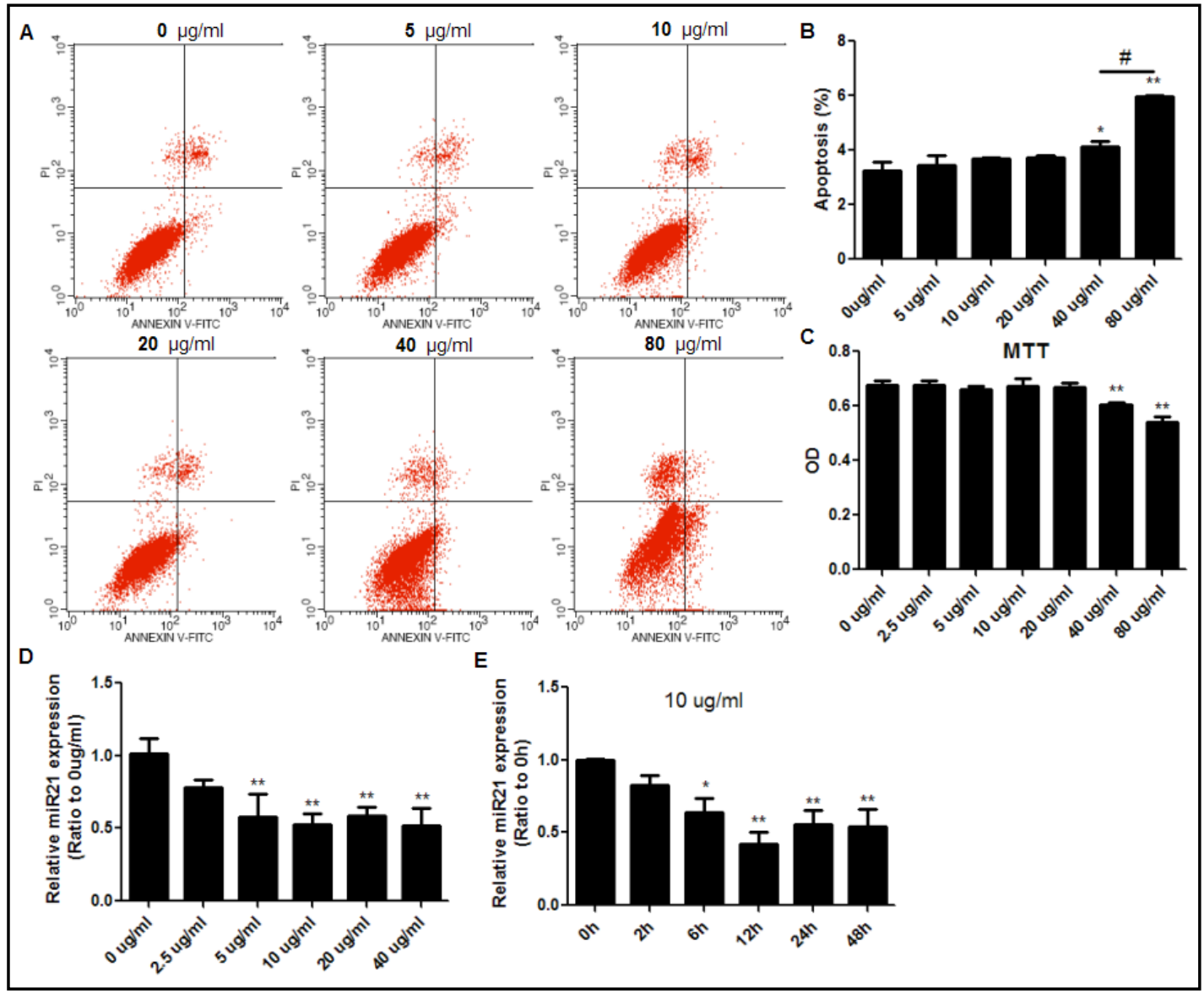

Fig. 2. Effects of TFs on cell apoptosis, viability, and miR-21 expression in mTECs. (A) Results of cell apoptosis assay by flow cytometry. mTECs were treated with TFs at indicated concentrations for 24 h. Q2 and Q3 areas indicate apoptotic cells. (B) Apoptosis rates. (C) Cell viability as assessed by a MTT assay. (D) miR-21 expression in mTECs treated with TFs at indicated concentrations for $24 \mathrm{~h}$. (E)miR-21 expression in mTECs treated with $10 \mu \mathrm{g} / \mathrm{mL}$ TFs for $2,6,12,24$ or $48 \mathrm{~h} .{ }^{*} \mathrm{p}<0.05$, ${ }^{*} \mathrm{p}<0.01$, compared with the $0 \mu \mathrm{g} / \mathrm{mL}$ or 0 h group; $\# \mathrm{p}<0.01$. 
$\mathrm{mL}$ (Fig. 2D). Furthermore, the TFs down-regulated miR-21 expression in a time-dependent manner and significantly reduced miR-21 expression $6 \mathrm{~h}$ after administration (Fig. 2D).

\section{TFs attenuated TGF- $\beta 1$-induced fibrosis via modulation of miR-21 and Smad signaling}

We used TGF- $\beta 1$ to induce fibrosis in vitro and investigated the effects of TFs on production of fibrotic markers in the mTECs. As shown in Fig. 3, both a low $(10 \mu \mathrm{g} / \mathrm{mL})$ and high $(40 \mu \mathrm{g} / \mathrm{mL})$ dose of TFs significantly down-regulated expression of miR-21 in TGF- $\beta 1$ treated mTECs, attenuating TGF- $\beta 1$-induced expression of Collagen I, Collagen III, and $\alpha$-SMA at the RNA and protein levels. Meanwhile, expression of Smad signaling was also observed in the mTECs, revealing marked up-regulation of Smad7 expression and suppression of Smad3 phosphorylation with TFs treatment (Fig. 3E). We also investigated the effect of TFs on expression of miR-21 and Smad signaling in vivo, revealing inhibition of miR-21 expression, up-regulation of Smad7 protein levels, and down-regulation of Smad3 phosphorylation in the kidneys at 14 days after UUO surgery (Fig. 4A, B).

To determine whether the anti-fibrotic effects conferred by the TFs were mediated by miR-21, mTECs were transfected with a miR-21 mimic prior to TFs treatment. As a result, overexpression of miR-21 enhanced expression of Collagen I, Collagen III, and $\alpha$-SMA compared with the control, abolishing the anti-fibrotic effects of TFs on TGF- $\beta 1$-treated mTECs. Moreover, the miR-21 mimic down-regulated Smad7, activating phosphorylation of Smad3 (Fig. 4C).

Deletion of miR-21 ameliorated renal fibrosis and up-regulated Smad7 expression in UUO mice

To determine the effects of miR-21 on renal fibrosis and expression of Smad signaling, we used miR-21\% mice and wild-type littermate controls in a UUO murine model. We found that, compared with wild-type littermates, miR-21\% mice showed milder tubulointerstitial fibrosis at 14 days after surgery, as demonstrated by Masson's trichrome staining (Fig. 5A). In addition, minor accumulation of Collagen I, Collagen III, and $\alpha$-SMA-positive cells was observed in the ligated kidneys of miR-21\% mice (Fig. 5B, C). Deletion of miR-21 in UUO kidneys significantly up-regulated expression of Smad7, inhibiting phosphorylation of Smad3 (Fig. 5D).

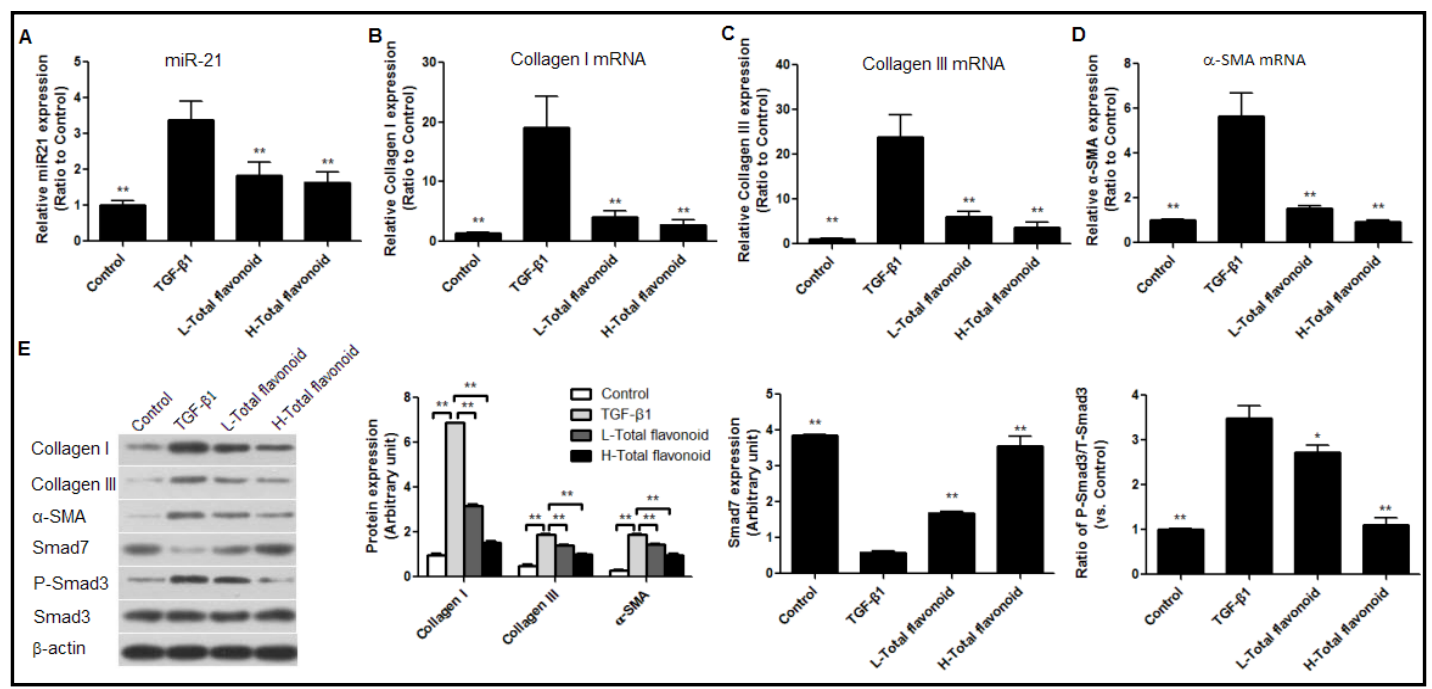

Fig. 3. TFs attenuated TGF- $\beta 1$ - induced fibrosis via modulation of miR-21 and Smad signaling. (A) miR-21 expression. (B) Collagen I mRNA expression. (C) Collagen III mRNA expression. (D) $\alpha$-SMA mRNA expression. (E) Protein levels of fibrotic markers and Smad signaling in TGF- $\beta 1$-induced mTECs treated with TFs. Data in E represent the mean from three independent experiments. L-Total flavonoid and H-Total flavonoid: low $(10 \mu \mathrm{g} / \mathrm{mL})$ and high dose $(40 \mu \mathrm{g} / \mathrm{mL})$ of TFs added to TGF- $\beta 1$-induced mTECs, respectively. ${ }^{*} \mathrm{p}<0.05$, ${ }^{* *} \mathrm{p}<$ 0.01 , compared with the TGF- $\beta 1$ group. 


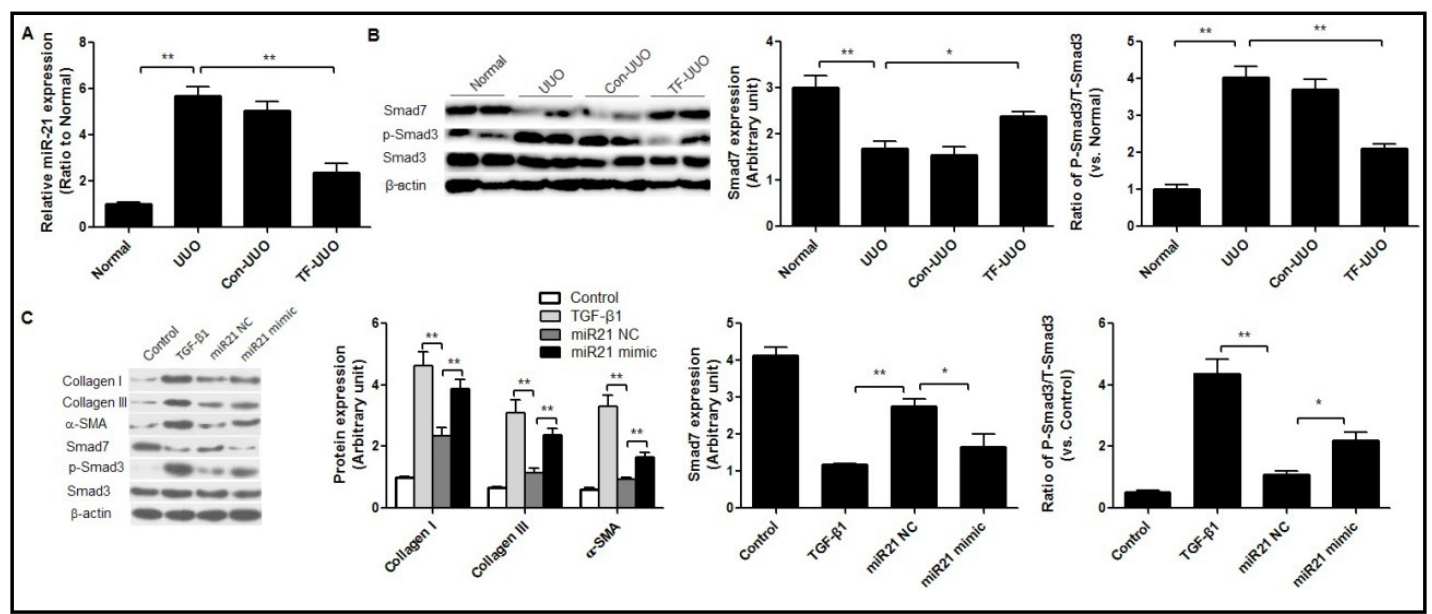

Fig. 4. TFs treatment inhibited Smad signaling and overexpression of miR-21 abolished the anti-fibrotic effects of TFs. (A) TFs treatment inhibited miR-21 expression in UUO mice. (B) TF treatment up-regulated Smad7 protein levels, and suppressed phosphorylation of Smad3 in UUO mice. (C) Results of western blot analysis. A miR-21 mimic was transfected into mTECs $48 \mathrm{~h}$ before TF $(10 \mu \mathrm{g} / \mathrm{mL})$ and TGF- $\beta 1(10 \mathrm{ng} / \mathrm{mL})$ treatment. miR-21 NC: miRNA mimic negative control, used as a control of the miR-21 mimic. Data in B and C represent the mean from three independent experiments. ${ }^{*} \mathrm{p}<0.05,{ }^{* *} \mathrm{p}<0.01$.

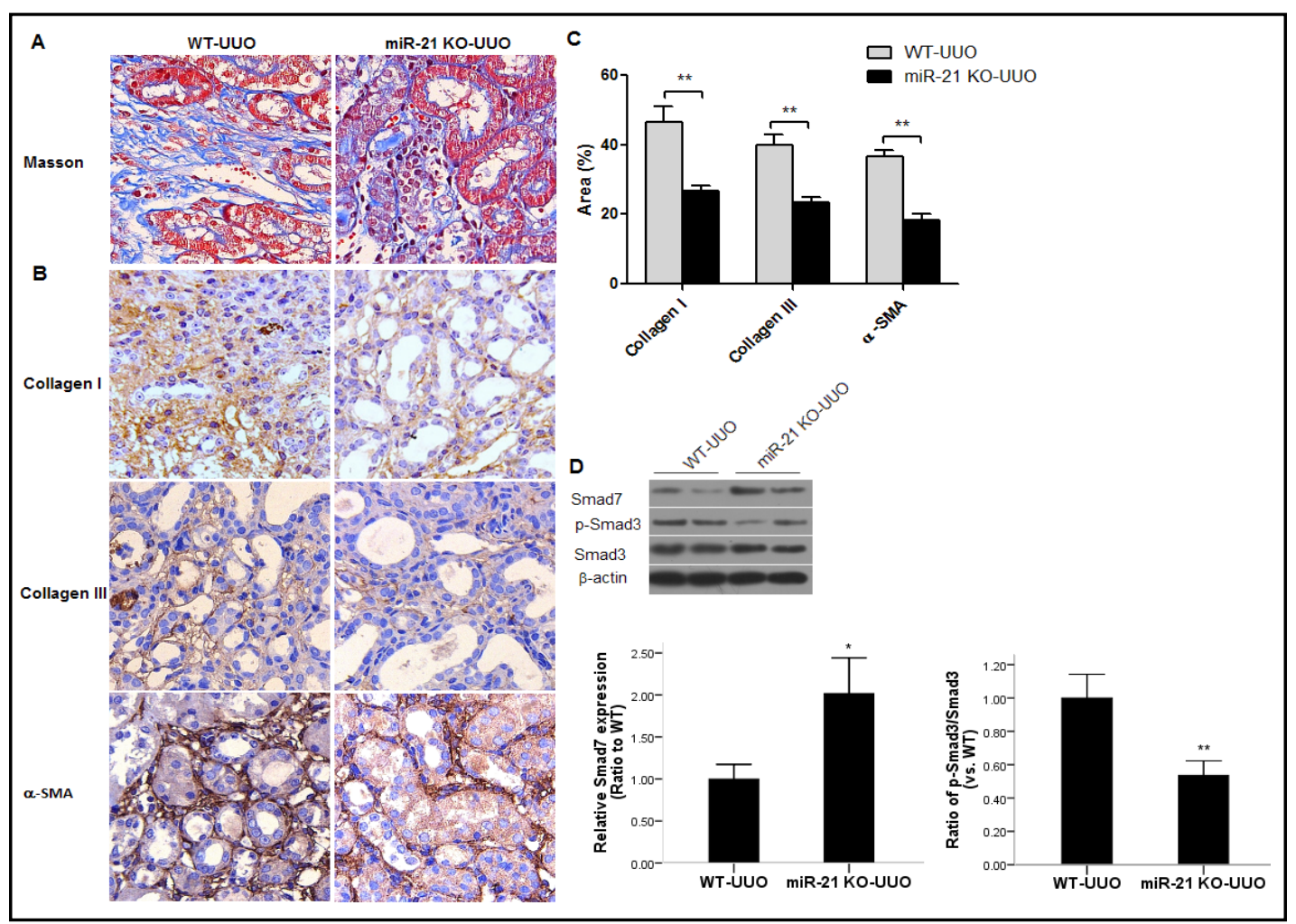

Fig. 5. Deletion of miR-21 ameliorated renal fibrosis and up-regulated Smad7 expression. (A) Masson trichrome staining of kidneys from miR-21\% mice and wild-type littermates subjected to UUO. Magnification: $\times 100$. (B) Immuno -histochemical staining of Collagen I, Collagen III, and $\alpha$-SMA. (C) Quantitative analysis of immunohistochemical staining. ${ }^{*} \mathrm{p}<0.05,{ }^{* *} \mathrm{p}<0.01$. (D) Expression of Smad signaling in kidneys of miR-21 ${ }^{*}$ 1- mice and wild-type littermates subjected to UUO was measured by western blotting. Data represent the mean from three independent experiments. ${ }^{*} \mathrm{p}<0.05,{ }^{* *} \mathrm{p}<0.01$. 


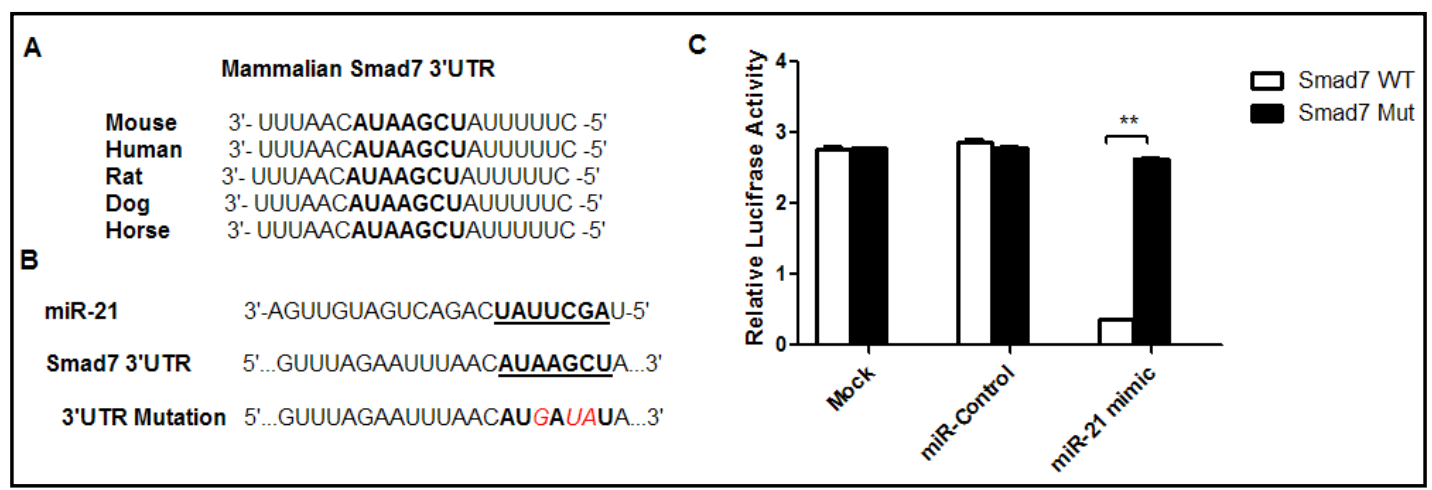

Fig. 6. miR-21 targets Smad7 mRNA in renal tubular epithelial cells. (A) Binding site of miR-21 located in the $3^{\prime}$-UTR of Smad7, which is very conserved in mammalian Smad7 transcripts. (B) Wild-type and mutated Smad7 3'-UTR. (C) Luciferase activity. mTECs were cotransfected with a miR-21 mimic or miRNA control, and reporter vectors containing wild-type (Smad7 WT) or mutated (Smad7 Mut) Smad7 3'-UTR. Luciferase activity was then measured. ${ }^{* *} \mathrm{p}<0.01$.

miR-21 targets Smad7 $m R N A$ in renal TECs

As demonstrated above, overexpression of miR-21 down-regulated Smad7 (Fig. 4C), while deletion of miR-21 up-regulated Smad7 expression in the kidneys (Fig. 5D). These findings suggest that miR-21 negatively regulates Smad7 expression in the kidneys. Using bioinformatics analysis with Target Scan Release 6.2, the binding site of miR-21 was located in the 3'-UTR of Smad7, which is very conserved in mammalian Smad7 transcripts (Fig. 6A, B). A dual-luciferase reporter assay was subsequently carried out to determine whether Smad7 is post-transcriptionally regulated by miR-21. As shown in Fig. 6C, luciferase activity significantly decreased when the reporter containing the Smad7 3'-UTR was co-transfected with a miR-21 mimic. In contrast, no decrease was observed in the reporter containing the mutated miR-21 binding site. These results suggest that miR-21 negatively regulates Smad7 expression by targeting the 3 '-UTR of Smad7.

\section{Discussion}

The prevalence of CKD is on the rise, and in China, the overall prevalence is currently $10.8 \%$, with the number of patients with CKD thought to be around 119.5 million [28]. Renal fibrosis is the final stage of CKD leading to loss of renal function $[29,30]$. In the present study, we investigated the effects of TFs on the progression of renal fibrosis in a mouse model of UUO. As a result, we found that the TFs reduced expression of miR-21 in the kidneys, upregulated Smad7 expression, the target gene of miR-21, and inhibited phosphorylation of Smad3, thereby attenuating renal fibrosis.

Activation of TGF- $\beta 1 /$ Smad signaling occurs in almost all forms of progressive CKD, playing a critical role in the onset and development of renal fibrosis [8,31]. Smad3, but not Smad2, is the key downstream mediator of TGF- $\beta 1$ responsible for renal fibrosis [15], while TGF- $\beta 1$ increases phosphorylation of Smad3 and decreases Smad7 expression. Activation of Smad3 can directly promote the expression of collagens, fibronectin, and $\alpha$-SMA, leading to renal fibrogenesis [11]. In addition, Smad3 activation was found to induce expression of Smad7, which then suppressed TGF- $\beta 1 /$ Smad3 signaling via negative feedback by acting as an inhibitory Smad [8], protecting the kidneys from renal fibrosis. Previous studies also revealed that Smad7 knockout mice exhibited enhanced renal TGF- $\beta 1 /$ Smad3 signaling and developed more severe renal fibrosis in both obstructive nephropathy and diabetic kidney disease [25, 32]. However, Smad7 treatment suppressed activation of TGF- $\beta 1 /$ Smad3 signaling, thereby ameliorating progressive forms of CKD such as obstructive nephropathy 
and crescentic glomerulonephritis [33, 34]. Consistent with previous reports, our results suggest that TGF- $\beta 1$ expression is significantly up-regulated in the kidneys of mice subjected to UUO. Meanwhile, phosphorylation of Smad3 was markedly increased and Smad7 expression decreased. Similar findings were also observed in TGF- $\beta 1$-treated mTECs.

TGF- $\beta 1 /$ Smad3 signaling also exerts a fibrotic effect by regulating miRNA expression in kidney disease [8]. The interaction between TGF- $\beta 1$ signaling and miRNAs during kidney disease has well been documented, with TGF- $\beta 1$-induced expression of miR-21, miR-192, and miR-433 known to participate in renal fibrogenesis $[35,36]$. However, TGF- $\beta 1$ signaling was also found to inhibit miR-29 and miR-200 family expression, protecting kidneys from renal fibrosis [37, 38]. TGF- $\beta 1$ regulates miR-21 expression via a Smad3-, but not Smad2, dependent mechanism during renal fibrosis [15]. Deletion of Smad3 inhibited TGF- $\beta 1$ induced miR-21expression in mouse embryonic fibroblasts and kidney TECs [15]. miR-21 is also able to activate TGF- $\beta 1$ signaling via downregulation of Smad7, acting in a feed-forward loop resulting in TGF- $\beta 1$ signaling amplification [14]. Phosphatase and tensin homolog (PTEN) is also a major target of mir-21 and participates in the regulation of Smad2/Smad3 phosphorylation and transcriptional activity [39]. Complex formation of PTEN with Smad2/ Smad3 was also found to induce Smad2/Smad3 dephosphorylation in the nucleus, inactivating TGF- $\beta 1$ signaling and subsequently inhibiting fibrosis. PTEN is also a negative regulator of the TGF- $\beta 1$ signaling pathway [40]. Meanwhile, miR-21 acts as an important suppressor of Smad- and Akt-dependent TGF- $\beta 1$ signaling pathways by targeting Smad7 and PTEN [41]. Previous studies have also demonstrated that miR-21 positively regulates production of ECM and $\alpha$-SMA in kidney cells, such as TECs and mesangial cells, after administration of TGF- $\beta 1[15,42]$. Persistent overexpression of miR-21 also contributes to renal fibrosis, while knockdown of miR-21 inhibits progression [15]. In this study, high expression of Smad3 phosphorylation and miR-21 was observed in TGF- $\beta 1$-treated mTECs and fibrotic kidneys from mice with UUO. miR-21 knockout mice exhibited an attenuated fibrotic response to UUO, as demonstrated by decreased ECM accumulation, and reduced expression of Collagen I, Collagen III, and $\alpha$-SMA compared with wild-type mice. Interestingly, the negative regulator of TGF- $\beta 1 /$ Smad3 signaling, Smad7, is targeted and suppressed by miR-21 in several diseases. For example, McClelland et al. [41] demonstrated that miR-21 is associated with the development of diabetic nephropathy, while targeting of Smad7 was found to be essential to the role of miR-21 in TGF- $\beta$-mediated fibrotic signaling. Overexpression of miR21 in TECs inhibited the TGF- $\beta 1$-induced increase in Smad7 protein. Furthermore, Liu et al. found that miR-21 mediated fibrogenic activation of pulmonary fibroblasts and lung fibrosis is mainly regulated by Smad7. In this study, elevated expression of Smad7 and reduced phosphorylation of Smad3 was observed in the kidneys of miR-21 knockout mice with UUO compared with wild-type mice. Combining bioinformatics analysis and a luciferase reporter assay, we identified and validated Smad7 as the target gene of miR-21 in TECs. Targeting miR-21 or maintaining Smad7 stability could therefore have therapeutic potential in the treatment of renal fibrosis.

TFs from different plantextracts have diverse functions. For example, C-glycosides isolated from Abrus mollis extracts possess anti-inflammatory activity, and have hepatoprotective effects in both acute and chronic liver injury [43]. Meanwhile, pretreatment with TFs from Scutellaria baicalensis stems and leaves was found to protect against cerebral ischemia reperfusion injury. The underlying mechanisms may involve inhibiting the apoptosis of hippocampal neurons via down-regulation of Fas and FasL protein expression, attenuating oxidative stress damage and increasing microvascular recanalization [44]. TFs extract from Abelmoschus manihot (L.) medic flowers was also found to protect mice against D-galactoseinduced oxidative stress in association with activation of Nrf2 signaling [45]. Meanwhile, in the present study, administration of TFs extracted from $C$. Cathayensis leaves attenuated renal fibrosis in a mouse model of UUO. Mechanistically, TFs suppressed the expression of miR-21, increased expression of the antifibrotic protein Smad7, the target gene of miR-21, and decreased phosphorylation of Smad3, thereby ameliorating TGF- $\beta 1$-induced collagen and $\alpha$-SMA expression. Furthermore, it is worth noting that PTEN, another important

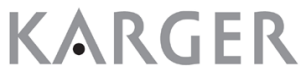


target of miR-21, might partly contribute to the anti-fibrotic effect of TFs; however, further investigation of this is required.

This study has some limitations that should be noted. Our study did not reveal the exact mechanism underlying TFs suppression of miR-21 expression in the kidneys. In addition, we did not identify the role of each TF component on progression of renal fibrosis. Further investigations are therefore needed to determine the effects of monomer extracts from $C$. Cathayensis leaves on renal fibrosis and its underlying mechanisms.

\section{Conclusion}

In conclusion, our study demonstrates the protective effect of TFs against renal tubulointerstitial fibrosis induced by UUO. The underlying mechanisms involve a TF-induced reduction in miR-21 expression, which regulates the downstream Smad signaling pathway, thereby attenuating expression of collagens and $\alpha$-SMA. TFs may be a novel and potential anti-fibrotic treatment for renal fibrosis.

\section{Acknowledgements}

This work was supported by the National Natural Science Foundation of China (81430015 to X.D. and 81471890 to P.J.), and the Science and Technology Commission of Shanghai Municipality (14DZ2260200, the Project of Shanghai Key Laboratory of Kidney and Blood Purification).

\section{Disclosure Statement}

The authors declare no conflicts of interest.

\section{References}

1 Ni L, Shi WY: Composition and free radical scavenging activity of kernel oil from Torreya grandis, Carya Cathayensis, and Myrica R ubra. Iran J Pharm Res 2014;13:221-226.

2 Wu W, Bi XL, Cao JQ, Zhang KQ, Zhao YQ: New antitumor compounds from Carya cathayensis. Bioorg Med Chem Lett 2012;22:1895-1898.

- 3 Tian SS, Jiang FS, Zhang K, Zhu XX, Jin B, Lu JJ, Ding ZS: Flavonoids from the leaves of Carya cathayensis Sarg. inhibit vascular endothelial growth factor-induced angiogenesis. Fitoterapia 2014;92:34-40.

4 Jin B, Ding Z: Flavonoids from Carya cathayensis Sarg. leaves inhibit carotid artery lesion formation induced by low blood flow. Biomed Pharmacother 2017;94:88-92.

-5 Tampe D, Zeisberg M: Potential approaches to reverse or repair renal fibrosis. Nat Rev Nephrol 2014;10:226-237.

-6 Zeisberg M, Kalluri R: Cellular mechanisms of tissue fibrosis. 1. Common and organ-specific mechanisms associated with tissue fibrosis. Am J Physiol Cell Physiol 2013;304:C216-C225.

7 Meng XM, Tang PM, Li J, Lan HY: TGF- $\beta$ /Smad signaling in renal fibrosis. Front Physiol 2015;6:82.

$>$ Lan HY: Diverse roles of TGF- $\beta$ /Smads in renal fibrosis and inflammation. Int J Biol Sci 2011;7:1056-1067.

-9 Nakao A, Imamura T, Souchelnytskyi S, Kawabata M, Ishisaki A, Oeda E, Tamaki K, Hanai J, Heldin CH, Miyazono K, ten Dijke P: TGF-beta receptor-mediated signalling through Smad2, Smad3 and Smad4. EMBO J 1997;16:5353-5362.

10 Feng XH, Derynck R: Specificity and versatility in Tgf-beta signaling through Smads. Annu Rev Cell Dev Biol 2005;21:659-693.

11 Lan HY, Chung AC: Transforming growth factor- $\beta$ and Smads. Contrib Nephrol 2011;170:75-82. 


\section{Cellular Physiology Cell Physiol Biochem 2018;49:1551-1563 \begin{tabular}{l|l} 
DOI: 10.1159/000493458 & $\begin{array}{l}\text { O } 2018 \text { The Author(s). Published by S. Karger AG, Basel } \\
\text { www.karger.com/cpb }\end{array}$
\end{tabular}}

12 Chung AC, Dong Y, Yang W, Zhong X, Li R, Lan HY: Smad7 suppresses renal fibrosis via altering expression of TGF- $\beta$ /Smad3-regulated microRNAs. Mol Ther 2013;21:388-398.

13 Jinek M, Doudna JA: A three-dimensional view of the molecular machinery of RNA interference. Nature 2009;457:405-412.

14 Patel V, Noureddine L: MicroRNAs and fibrosis.Curr Opin Nephrol Hypertens 2012;21:410-416.

15 Zhong X, Chung AC, Chen HY, Meng XM, Lan HY: Smad3-mediated upregulation of miR-21 promotes renal fibrosis. J Am Soc Nephrol 2011;22:1668-1681.

-16 Zarjou A, Yang S, Abraham E, Agarwal A, Liu G: Identification of a microRNA signature in renal fibrosis: role of miR-21. Am J Physiol Renal Physiol 2011;301:F793-F801.

17 Chau BN, Xin C, Hartner J, Ren S, Castano AP, Linn G, Li J, Tran PT, Kaimal V, Huang X, Chang AN, Li S, Kalra A, Grafals M, Portilla D, MacKenna DA, Orkin SH, Duffield JS: MicroRNA-21 promotes fibrosis of the kidney by silencing metabolic pathways. Sci Transl Med 2012;4:121ra18.

-18 Yuan J, Chen H, Ge D, Xu Y, Xu H, Yang Y, Gu M, Zhou Y, Zhu J, Ge T, Chen Q, Gao Y, Wang Y, Li X, Zhao Y: Mir-21 promotes cardiac fibrosis after myocardial infarction via targeting smad7. Cell Physiol Biochem 2017;42:2207-2219.

19 Thum T, Gross C, Fiedler J, Fischer T, Kissler S, Bussen M, Galuppo P, Just S, Rottbauer W, Frantz S, Castoldi M, Soutschek J, Koteliansky V, Rosenwald A, Basson MA, Licht JD, Pena JT, Rouhanifard SH, Muckenthaler MU, Tuschl T, et al.: MicroRNA-21 contributes to myocardial disease by stimulating MAP kinase signalling in fibroblasts. Nature 2008;456:980-984.

-20 Zhang Z, Zha Y, Hu W, Huang Z, Gao Z, Zang Y, Chen J, Dong L, Zhang J: The autoregulatory feedback loop of microRNA-21/programmed cell death protein 4/activation protein-1 (MiR-21/PDCD4/AP-1) as a driving force for hepatic fibrosis development. J Biol Chem 2013;288:37082-37093.

-21 Liu G, Friggeri A, Yang Y, Milosevic J, Ding Q Thannickal VJ, Kaminski N, Abraham E: miR-21 mediates fibrogenic activation of pulmonary fibroblasts and lung fibrosis. J Exp Med 2010;207:1589-1597.

-22 Godwin JG, Ge X, Stephan K, Jurisch A, Tullius SG, Iacomini J: Identification of a microRNA signature of renal ischemia-reperfusion injury. Proc Natl Acad Sci USA 2010;107:14339-14344.

23 Jia P, Wu X, Dai Y, Teng J, Fang Y, Hu J, Zou J, Liang M, Ding X: MicroRNA-21 Is required for local and remote ischemic preconditioning in multiple organ protection against sepsis. Crit Care Med 2017;45:e703-e710.

24 Jia P, Teng J, Zou J, Fang Y, Wu X, Liang M, Ding X: Xenon protects against septic acute kidney injury via miR21 target signaling pathway. Crit Care Med 2015;43:e250-e259.

25 Chung AC, Huang XR, Zhou L, Heuche IR, LaiK N, Lan HY: Disruption of the Smad7 gene promotes renal fibrosis and inflammation in unilateral ureteral obstruction (UUO) in mice. Nephrol Dial Transplant 2009;24:1443-1454.

-26 Liu Y, Taylor NE, Lu L, Usa K, Cowley AW Jr, Ferreri NR, Yeo NC, Liang M: Renal medullary microRNAs in Dahl salt-sensitive rats: miR-29b regulates several collagens and related genes. Hypertension 2010;55:974982.

27 Jia P, Teng J, Zou JZ, Fang Y, Zhang X, Bosnjak ZJ, Liang M, Ding X: miR-21 contributes to xenon-conferred amelioration of renal ischemia-reperfusion injury in mice. Anesthesiology 2013;119:621-630.

-28 Zhang L, Wang F, Wang L, Wang W, Liu B, Liu J, Chen M, He Q Liao Y, Yu X, Chen N, Zhang JE, Hu Z, Liu F, Hong D, Ma L, Liu H, Zhou X, Chen J, Pan L, et al.: Prevalence of chronic kidney disease in China: a crosssectional survey. Lancet 2012;379:815-822.

-29 Grande MT, Lopez-Novoa JM: Fibroblast activation and myofibroblast generation in obstructive nephropathy. Nat Rev Nephrol 2009;5:319-328.

-30 Zeisberg M, Neilson EG: Mechanisms of tubulointerstitial fibrosis. J Am Soc Nephrol 2010;21:1819-1834.

-31 Yeh YC, Wei WC, Wang YK, Lin SC, Sung JM, Tang MJ: Transforming growth factor- $\beta 1$ induces Smad3dependent $\beta 1$ integrin gene expression in epithelial-to-mesenchymal transition during chronic tubulointerstitial fibrosis. Am J Pathol 2010;177:1743-1754.

-32 Chen HY, Huang XR, Wang W, LiJ H, Heuchel RL, Chung AC Lan HY: The protective role of Smad7 in diabetic kidney disease: mechanism and therapeutic potential. Diabetes 2011;60:590-601.

33 Ka SM, Huang XR, Lan HY, Tsai PY, Yang SM, Shui HA, Chen A: Smad7 gene therapy ameliorates an autoimmune crescentic glomerulonephritis in mice. J Am Soc Nephrol 2007;18:1777-1788.

-34 Hou CC, Wang W, Huang XR, Fu P, Chen TH, Sheikh-Hamad D, Lan HY: Ultrasound-microbubble-mediated gene transfer of inducible Smad7 blocks transforming growth factor-beta signaling and fibrosis in rat remnant kidney. Am J Pathol 2005;166:761-771. 


\section{Cellular Physiology Cell Physiol Biochem 2018;49:1551-1563 \begin{tabular}{l|l|l} 
DOI: 10.1159/000493458 & $\begin{array}{l}\text { O } 2018 \text { The Author(s). Published by S. Karger AG, Basel } \\
\text { www.karger.com/cpb }\end{array}$ \\
\hline
\end{tabular}}

-35 Chung AC, Huang XR, Meng X, Lan HY: miR-192 mediates TGF-beta/Smad3-driven renal fibrosis. J Am Soc Nephrol 2010;21:1317-1325.

-36 Li R, Chung AC, Dong Y, Yang W, Zhong X, Lan HY: The microRNA miR-433 promotes renal fibrosis by amplifying the TGF- $\beta /$ Smad3-Azin1 pathway. Kidney Int 2013;84:1129-1144.

-37 Qin W, Chung AC, Huang XR, Meng XM, Hui DS, Yu CM, Sung JJ, Lan HY: TGF- $\beta /$ Smad3 signaling promotes renal fibrosis by inhibiting miR-29. J Am Soc Nephrol 2011;22:1462-1474.

-38 Xiong M, Jiang L, Zhou Y, Qiu W, Fang L, Tan R, Wen P, Yang J: The miR-200 family regulates TGF- $\beta 1$-induced renal tubular epithelial to mesenchymal transition through Smad pathway by targeting ZEB1 and ZEB2 expression. Am J Physiol Renal Physiol 2012;302:F369-F379.

39 Bu S, Kapanadze B, Hsu T, Trojanowska M: Opposite effects of dihydrosphingosine 1-phosphate and sphingosine 1-phosphate on transforming growth factor-beta/Smad signaling are mediated through the PTEN/PPM1A-dependent pathway. J Biol Chem 2008;283:19593-19602.

-40 White ES, Atrasz RG, Hu B, Phan SH, Stambolic V, Mak TW, Hogaboam CM, Flaherty KR, Martinez FJ, Kontos CD, Toews GB: Negative regulation of myofibroblast differentiation by PTEN (Phosphatase and Tensin Homolog Deleted on chromosome 10). Am J Respir Crit Care Med 2006;173:112-121.

41 McClelland AD, Herman-Edelstein M, Komers R, Jha JC, Winbanks CE, Hagiwara S, Gregorevic P, Kantharidis P, Cooper ME: miR-21 promotes renal fibrosis in diabetic nephropathy by targeting PTEN and SMAD7. Clin Sci (Lond) 2015;129:1237-1249.

42 Zhong X, Chung AC, Chen HY, Dong Y, Meng XM, Li R, Yang W, Hou FF, Lan HY: miR-21 is a key therapeutic target for renal injury in a mouse model of type2 diabetes. Diabetologia 2013;56:663-674.

43 Chen M, Wang T, Jiang ZZ, Shan C, Wang H, Wu MJ, Zhang S, Zhang Y, Zhang LY: Anti-inflammatory and hepatoprotective effects of total flavonoid C-glycosides from Abrus mollis extracts. Chin J Nat Med 2014;12:590-598.

44 Kong X, Kong W, Miao G, Zhao S, Chen M, Zheng X, Bai J: Pretreatment with scutellaria baicalensis stemleaf total flavonoid protects against cerebral ischemia/reperfusion injury in hippocampal neurons. Neural Regen Res 2014;9:2066-2073.

45 Qiu Y, Ai PF, Song JJ, Liu C, Li ZW: Total flavonoid extract from Abelmoschus manihot (L.) medic flowers attenuates D-galactose-induced oxidative stress in mouse liver through the Nrf2 pathway. J Med Food 2017;20:557-567. 